\title{
The single particle tracking system
}

\author{
Ai-Tang Chang ${ }^{\mathrm{a}}$, Yi-Ren Chang ${ }^{\mathrm{b}}$, Sien $\mathrm{Chi}^{\mathrm{b}, \mathrm{c}}$, and Long $\mathrm{Hsu}^{\mathrm{a}}$ \\ ${ }^{a}$ Department of Electrophysics, National Chiao Tung University \\ ${ }^{\mathrm{b}}$ Institute of Eletro-Optical Engineering, National Chiao Tung University \\ ${ }^{\mathrm{c}}$ Department of Electrical Engineering, Yuan Ze University \\ 1001 TaHsueh Road, Hsinchu, Taiwan 300, ROC \\ 886-3-5712121\#56165, howard.ep91g@nctu.edu.tw
}

\begin{abstract}
In an optical tweezers system, the force measurement with a resolution less than pico-Newton can be achieved by precise measurement and analysis of the trapped particle trajectory. Typically, this single particle tracking technique is realized by a quadrant position sensor which detects the scattering lights of the trapping laser beam from the trapped particle. However, as the radius of the trapped particle is larger than the wavelength of the trapped laser, the scattering pattern becomes complicated, and it limits the tracking region and the signal sensitivity on the trapped particle. To solve this issue, an extra probing laser with optimized focal offset according to the trapping laser is applied to improve the flexibility and performance of our particle tracking system for each particle size. A rule of thumb between the optimized focal offsets and particle size is also concluded from the experimental results and theoretical simulations.
\end{abstract}

Keyword: photonic force microscopy; quadrant-photo detector; particle tracking.

\section{Introduction}

The force measurement, within a microscopic observation region, is an important issue in many fields such as biophysics, condensed material physics, and hydrodynamics of micro-sized system. Since 1996, E. H. f. Stelzer developed the photonic force microscope (PFM) ${ }^{1}$. PFM has become a powerful tool to analysis and investigate the mechanical forces of microstructures in microscopy system. A typical PFM setup is combined two parts —— a probe particle which is a micro-sized polystyrene bead trapped by an optical tweezers and a position sensor system to measure the displacement of the trapping particle with respect to the trapping laser focus. To take a biomechanical application as an example, a probe particle is tethered to the cell or molecule under study. Around the trapping laser focus, the optical tweezers provides a restoring force on the probe particle to the trapping center and the force is linear in the probe particle displacement. Consequently, the measurement of an external force is reduced to measuring the position of the probe particle in the calibrated optical tweezers system around the trapped center.

Optical Trapping and Optical Micromanipulation VII, edited by Kishan Dholakia, Gabriel C. Spalding, Proc. of SPIE Vol. 7762, 77622Q - (c) 2010 SPIE · CCC code: 0277-786X/10/\$18 - doi: 10.1117/12.860934 
As illustrated in Fig. 1, the schematic setup of the position sensor system is used to measure the probe particle position. The focus of the trap laser, converged by the objective, captures the probe particle. The condenser (CL) collects the forward scattering light from the trap laser on the quadrant-photo detector (QPD1), and the backward scattering light, collected by the objective, illuminates on the other quadrant-photo detector (QPD2). When the trapped particle slightly moves from the trapping center, the scattering light also changes on the quadrant-photo detectors QPD1 and QPD2. The three dimensional position of the probe particle can be measured through the scattering light variation. A quadrant-photo detector compares four photo sensors. Along the $\mathrm{x}$ direction, the displacement of the particle $b=\left(b_{x}, b_{y}, b_{z}\right)$ can be measurement from the signal $S_{x}=[(B+D)-(A+C)] /$ $\left[A^{\prime}+B^{\prime}+C^{\prime}+D\right]$ in Fig. 1 (d). For the same reason, the signal $S_{y}=[(A+B)-(C+D)] /\left[A^{\prime}+B^{\prime}+C^{\prime}+D^{\prime}\right]$ is measured for the displacement of the particle along the y direction and the signal $S_{z}=(A+B+C+D) /$ $\left[A^{\prime}+B^{\prime}+C^{\prime}+D^{\prime}\right]$ is measured for the displacement along the $z$ direction. The normalized factor $\left[A^{\prime}+B^{\prime}+C^{\prime}+D^{\prime}\right]$ is defined the signal measured by the quadrant-photo detector when the particle is captured at the trapping center.

(a)

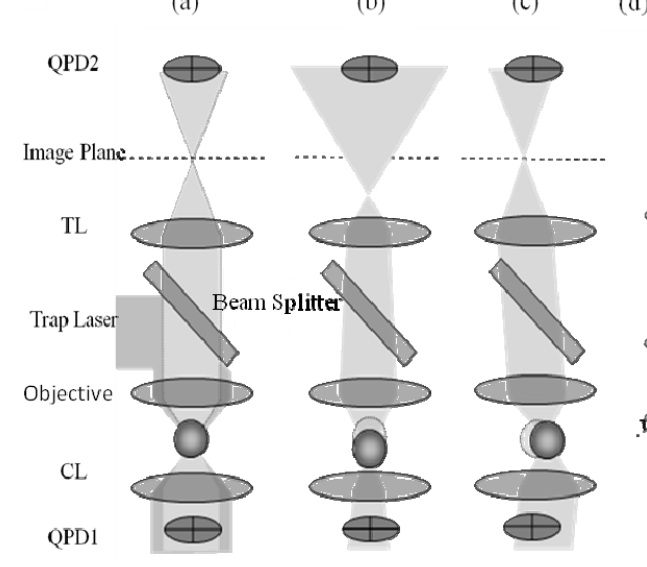

(d)

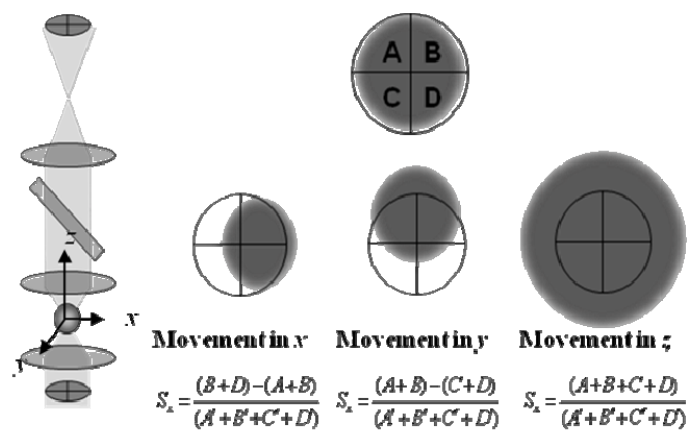

Fig. 1.The schematic setup of the position sensor system

However, the scattering pattern is complicated on the position sensor, when the radius of the probe particle is larger than the laser wavelength. The linear range of the displacement signal is reduced. Otherwise, the three dimensional signals are not independent on each direction. In other words, the signal $S_{x}$ not only varies with the particle movement along the x direction but also changes with the particle movement along another direction. To solve this problem, an extra laser source, rather than the trap laser, is used to detect the movement of the trapped particle. We call this optical mechanism as the single particle tracking system. To adjust the focal offset of the probe laser, the linear range and the sensitivity of the quadrant-photo detector signals will be enhanced effectively and the signal coupling effect is also reduced. The relationship between optimized focal offset of the probe laser and the 
tracked particle size is obtained experimentally and theoretically $2,3,4$ and 5 .

\section{Setup}

Fig. 2 shows the schematic representation of the optical setup. The trap laser source is Nd:YAG solid state laser emitting a laser beam at the wavelength of $800 \mathrm{~nm}$ in water. A beam expander (Lens L1 and tube lens TL) makes the trap laser to overfill the entrance pupil of the objective (Obj.) in order to optimize the optical tweezers efficient. A 100X oil-immersed objective with a numerical aperture of 1.25 serves as the focal lens. The trapping laser beam, after the objective, is focused at the objective optical axis to trap a micro-sized spherical particle. We use a piezo-translation stage (PZT), controlled by a computer, as the sample holder to move the sample precisely. The Minimum step of this stage is $1.22 \mathrm{~nm}$.

As for the single particle tracking system, a helium-neon $(\mathrm{He}-\mathrm{Ne})$ laser, with the wavelength of $475.8 \mathrm{~nm}$ in water, is used as the probe laser in our experimental setup. A lens set (L4 and L5) also makes the probe laser beam to overfill the entrance pupil of the objective. The lens L4 is movable to control the focal position of the probe laser beam on the objective optical axis. A condenser (CL) collects the forward scattering light of the probe laser from the trapped particle, directing the light on the quadrant photo-detector (QPD1). The condenser with the numerical aperture of 0.65 is co-focal with the objective ${ }^{6}$ and QPD1 is placed at the backward focal plane of the condenser. Additionally, the backward scattering light, sticking on the other quadrant photo-detector (QPD2) from the beam splitter (BS), is collected by the objective. QPD2 is placed near the image plane of the objective and lens L3. To reduce the signal noises of the quadrant photo-detectors, the filters are placed before these two detectors to prevent from the incidence of the trapping laser beam. A CCD camera is used to monitor the image of the sample.

Proc. of SPIE Vol. 7762 77622Q-3 


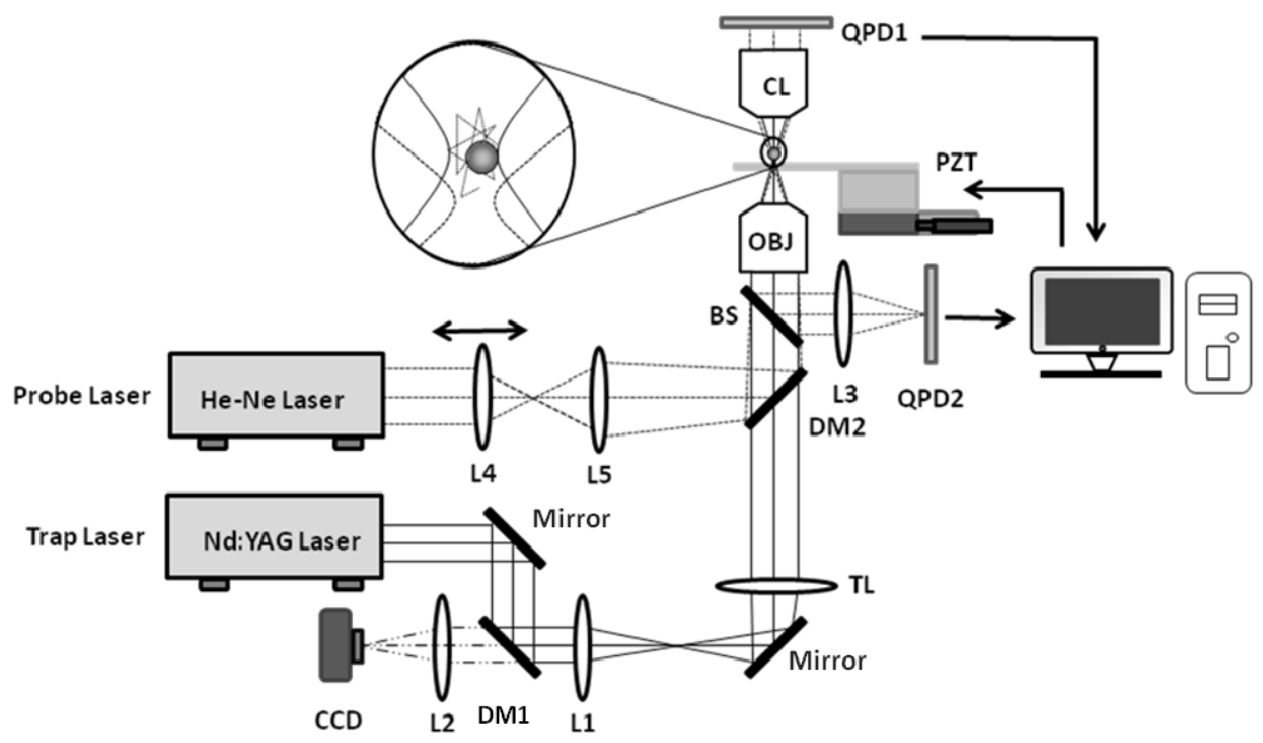

Fig. 2 shows the optical setup of the single particle tracking system. (DM: dichoric mirror; TL: tube lens; CL: condenser; BS: beam splitter; QPD: quadrant-photo detector)

\section{Method}

To accurately control the focal position of the probe laser, the piezo-translation stage is used to be a

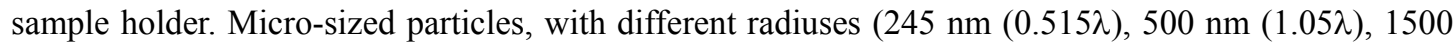
$\mathrm{nm}(3.15 \lambda), 2200 \mathrm{~nm}(4.62 \lambda)$ and $3100 \mathrm{~nm}(6.52 \lambda))$, are fixed in an agarose gel (1\% agarose aqueous) on the piezo-translation stage. These particles are moved according to the immobile focal position of the probe laser. There are two remarkable features in this experimental method. At first, to change the probe laser focal point along the objective optical axis, the adjustment of the optical mechanism brings some errors with a slight lateral displacement of the focal point from the optical axis. In place of the adjustment of the optical mechanism with the errors, the piezo-translation stage provides the precise movement to achieve the stable experiment platform.

Further, the spherical particles is fixed in the agarose gel rather than be adhered on a microscope glass slide. The agarose gel is made by mixing $1 \%$ agarose powders with $99 \%$ deionized water. The refraction index of the gel is very close to the deionized water. Thus, the gel with a fixed spherical particle is very similar to the conditions of the spherical particle which is trapped by optical tweezers in the water, and the particle does not randomly move due to Brownian motion.

As illustrated in Fig. 3(a), the spherical particle, fixed in the gel on the piezo-translation stage, is moved step by step at the $x-y$ plane perpendicular the optical axis ( $z$ axis). The displacement signals of 
the quadrant-photo detectors, used to detect the forward and backward scattering light of the probe laser from the particle, are recorded for each step. The distance of each step is $24.4 \mathrm{~nm}$, and the total movement area of the particle is 2440 X $2440 \mathrm{~nm}^{2}$ ( $5 \lambda$ X $5 \lambda$ ). After the signals recording finished at one layer, the particle is moved to next layer along the optical axis to execute the signal recording process continuously. According to each particle size, the total displacement range and each step of the piezo-translation stage, along the optical axis, are also different.

\section{Results}

The spherical particle is a symmetric shape and a uniform material. When the particle is moved across the optical axis on the $x-y$ plane, the signals, detected by the quadrant-photon detectors, are also symmetric profiles. The symmetry property of the quadrant-photon detector signals $\left(S_{x}, S_{y}\right.$, and $\left.S_{z}\right)$ can be shown as:

$$
\begin{gathered}
S_{x}\left(b_{x}, b_{y}\right)=-S_{x}\left(-b_{x}, b_{y}\right)=S_{x}\left(b_{x},-b_{y}\right) \\
S_{y}\left(b_{x}, b_{y}\right)=-S_{y}\left(-b_{x}, b_{y}\right)=S_{y}\left(b_{x},-b_{y}\right) \\
S_{z}\left(b_{x}, b_{y}\right)=S_{z}\left(-b_{x}, b_{y}\right)=S_{z}\left(b_{x},-b_{y}\right) .
\end{gathered}
$$

On the $x-y$ plane, the symmetric centers of the quadrant-photon detector signals are detected when the particle is right at the optical axis. Thus, we can define this symmetric center signal as the origin point, and find out the origin point in each $x-y$ layer. As illustrated in the Fig. 3, the signals $\left(S_{x}\right.$ and $\left.S_{y}\right)$, detected by QPD1 (forward scattering light), are the function of the particle displacements $\left(b_{x}\right.$ and $\left.b_{y}\right)$ at two different layers $\left(b_{z l}\right.$ and $\left.b_{z 2}\right)$. The radius of the spherical particle is $0.5 \mu \mathrm{m}(1.05 \lambda)$. The contour maps $(\sim 3 \lambda \times 3 \lambda)$ are cut off the section of the raw experiment date $(5 \lambda \times 5 \lambda)$, and the origin point is the center point of the each contour map.

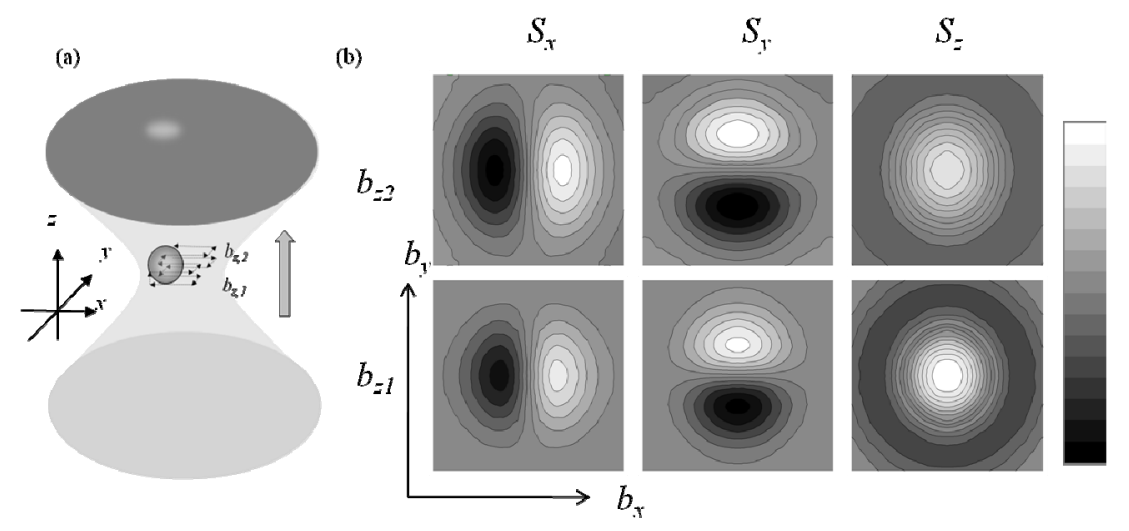

Fig. 3. The signals $\left(S_{x}\right.$ and $S_{y}$ ), detected by QPD1 (forward scattering light), are the function of the particle 
displacements $\left(b_{x}\right.$ and $\left.b_{y}\right)$ at two different layers $\left(b_{z 1}\right.$ and $\left.b_{z 2}\right)$.

In addition, the particle, on the optical axis, moved upward the distance $b_{z}$ according to the fixed probe laser focus is equal to downward the same distance $b_{z}$ of the probe laser focus from the immovable particle. The focal displacement of the probe laser $Z_{p}$ can be defined as the focal position according to the altitude of the tracked particle along the optical axis $\left(Z_{p}=-b_{z}\right)$. The quadrant-photon detector signals, along the optical axis, can be obtained from each x-y layer. As illustrated in Fig. 4, the quadrant-photon signals $S_{x}$ and $S_{z}$, along the optical axis, are the function of the particle displacement $b_{x}$ and the displacement of the probe laser focus $Z_{p}$.

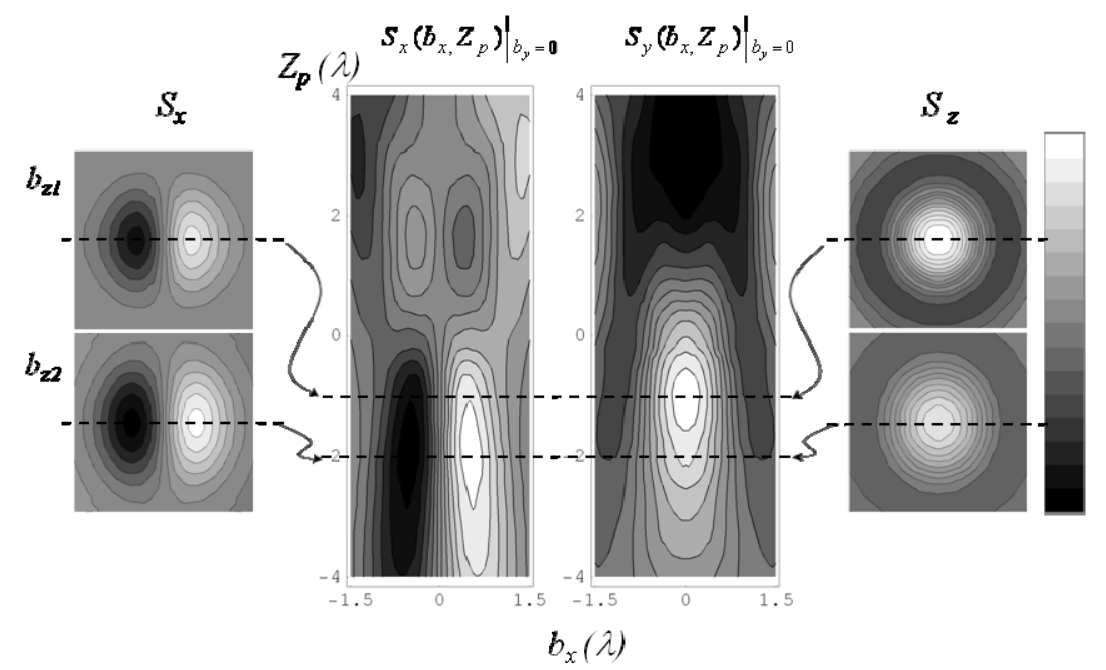

Fig. 4. The quadrant-photon signals $S_{x}$ and $S_{z}$, along the optical axis, are the function of the particle displacement $b_{x}$ and the probe laser focal displacement $Z_{p}$.

From this experimental result, an optical tweezers with a suitable particle tracking system can be built. The suitable displacement of the probe laser focus is about $2 \lambda(\sim 950 \mathrm{~nm})$, when the particle, with the radius $0.5 \mu \mathrm{m}$, is tracked in the optical tweezers system with forward scattering configuration. The trapping laser focus is regulated at the origin point in Fig. 4, since the particle is usually trapped around the focal point of the trapping laser. Therefore, the coupling influence of the quadrant-photo detector signals will be effective reduction.

Otherwise, the different size spherical particles, with radius $245 \mathrm{~nm}(0.515 \lambda), 500 \mathrm{~nm}(1.05 \lambda), 1500$ $\mathrm{nm}(3.15 \lambda), 2200 \mathrm{~nm}(4.62 \lambda)$, and $3100 \mathrm{~nm}(6.52 \lambda)$, have been measured by the configurations of the forward and backward scattering in our experiment. According to the experimental results, all the linear relationships, between the signals of the quadrant-photo detector and the particle displacements, can be found when the probe laser focus is located at the optimized position. 
To track a single particle position by using the quadrant-photon detector, the most important parameters are the signal sensitivity and the detectable tracking range of the particle displacement. Thus, these two parameters determine the optimized focal position of the probe laser. Considering the particle trapped around the trapping beam focus, the signal sensitivity of the particle displacement can be defined as:

$$
\vec{V}\left(Z_{p}\right)=\left.\left(\frac{\partial S_{x}}{\partial b_{x}}, \frac{\partial S_{y}}{\partial b_{y}}, \frac{\partial S_{z}}{\partial b_{z}}\right)\right|_{\vec{b}=(0,0,0)}
$$

or

$$
\vec{V}\left(Z_{p}\right)=\left.\left(\frac{\partial S_{x}}{\partial b_{x}}, \frac{\partial S_{y}}{\partial b_{y}},-\frac{\partial S_{z}}{\partial b_{z}}\right)\right|_{\vec{b}=(0,0,0)},
$$

where $\vec{V}\left(Z_{p}\right)$ is the signal sensitivity of the particle displacement. $S_{x}, S_{y}$, and $S_{z}$ are the quadrant-photo detector signals in the three dimensions and the coordinate $\vec{b}(b x, b y, b z)$ is particle position.

As for the detectable tracking range of the particle displacement, there are two important conditions. At first, the signal response of the quadrant-photo detector and the particle displacement are a linear relationship. After that, the signal, along one axis, is independent on another axis signal. Thus, the maximum detectable tracking range of the particle displacement $b_{i, \max }\left(Z_{p}\right)$ conforms to the following equations:

$$
E=\frac{\left|S_{i}\left(b_{i}, Z_{p}\right)\right|_{b_{j}=0}-b_{i} V_{i}\left(Z_{p}\right) \mid}{\left.S_{i}\left(b_{i}, Z_{p}\right)\right|_{b_{j}=0}} \leq 5 \% \text {, }
$$

and

$$
C=\frac{\partial S_{i}\left(b_{i}, Z_{p}\right) / b_{j}}{\partial S_{i}\left(b_{i}, Z_{p}\right) / b_{i}} \leq 5 \%, \text { where } i \neq j
$$

Where the indices $i$ and $j$ are $x, y$ and $z . E$ is the linear error, and $C$ is the signal coupling rate. The linear error is defined the ratio of the difference between the experimental signal and the linear value to the experimental signal. From the Eq. 6 and Eq. 7, the detectable tracking range of the particle displacement is not only determined by accepting the maximum linear error of $5 \%$, but also the maximum of the acceptable signal coupling rate is $5 \%$.

Proc. of SPIE Vol. 7762 77622Q-7 
The signal sensitivity and the detectable tracking range of the particle displacement have been defined. These two parameters are the important decisive factors of the optimized focal offset. Therefore, we define the working region $\vec{W}$ as:

$$
\vec{W}=\left(V_{x} b_{x, \max }, V_{y} b_{y, \max }, V_{z} b_{z, \max }\right),
$$

According to the maximum working region, the optimized focal position is decided and the position of the particle is measured with the sensitive signal responses of the quadrant-photo detector in three dimensions. As illustrated in Fig. 5, we have calculated the optimized focal offsets of the probe laser with respect to different size particles. The quadrant-photo detector signals, in three dimensions, were measured in the forward scattering configuration (a) (c) and the backward scattering configuration (d) $\sim($ f). The mark black squares $($ ) indicate the experimental data and the mark black spots $(\bullet)$ with the gray straight line are the theoretical simulations.

The experiment results are matched the theoretical simulations closely and the error of the optimized focal offset between experiment data and theoretical values are within hundreds of nanometers. Additionally, the optimized focal offset is very small when the radius of the particle is less than the wavelength of the probe laser. In other words, the trapping beam can be directly treated as the probe laser. When the radius of the tracked particle, however, is larger than the probe laser wavelength, the optimized focal offset cannot be ignored. An extra laser source is requested to track the particle.

Further, the optimized focal offset is larger with the increasing of the particle size. The relationship between the optimized focal offset and the particle size can be approximately estimated a linear equation. The optimized focal offset, within the focus of the trapping beam and the objective lens, is about 3.3 times of the particle radius from the focus of the trapping beam in the forward scattering confirmation. As for the backward scattering configuration, the optimized focal offset, between the trapping beam focus with the condenser lens, is about twice the length of the particle radius according to the trapping beam focus. The results provide a convenient way to adjust the probe laser position with tracking different size of the particles in the optical tweezers system. 

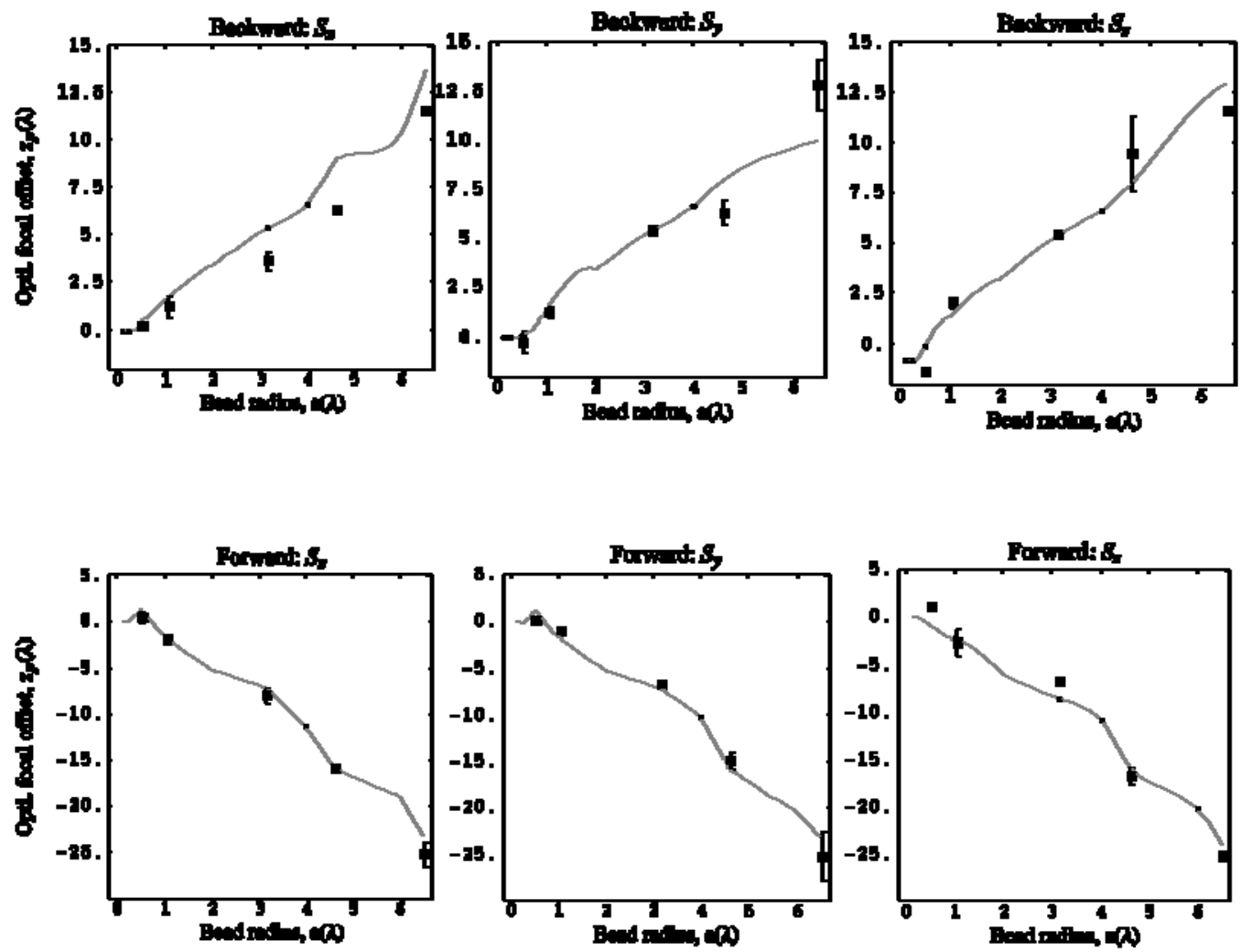

Fig. 5.The optimized focal offset with different sizes of the particles were obtained from the experimental data (the mark and theoretical simulations (gray straight line and the mark $\bullet$ ). (a) (c) are for the forward scattering configuration and (d) (f) are for the backward scattering configuration.

\section{Conclusions}

The optimized focal offsets according to different size trapped particles were obtained. When the focus of the probe laser is regulated at the optimized focal position, the signal coupling effect is reduced obviously. Simultaneously, the detectable tracking region and the signal sensitivity of particle displacement are enhanced effectively. Both the linear error and the signal coupling rate are less than $5 \%$. From experimental results and theoretical simulations, the optimized focal offset is larger with the increase of the particle size. The optimized focal position is located at the 3.3 times of the radius of the tracked particle between the focus of the trapping beam with the objective lens and according to the trapping beam focus in the forward scattering configuration. As for the backward scattering configuration, the optimized focal position is located at 2 times of the tracked particle radius according to the trapping beam focus and between the trapping beam focus with the condenser lens. It is easy way to help us to build the single particle tracking system with the off focused probe laser. 


\section{Reference}

1. E. L. Florin, J. K. H. Hörber, and E. H. K. Stelzer, "High-resolution axial and lateral position sensing using two-photon excitation of fluorophores by a continuous-wave Nd:YAG laser," Appl. Phys. Lett. 69 446-448 (1996).

2. H. C. van de Hulst, "Mie's formula solution," in Light scattering by small particles (Dover Publications, New York, 1981), pp.119-128.

3. A. Rohrbach and E. H. K. Stelzer, "Trapping force, force constants, and potential depth for dielectric sphere in the presence of spherical aberrations," Appl. Opt. 41, 2494-2507 (2002).

4. F. Gittes and C. Schmidt, "Signal and noise in micromechanical measurements,"Meth. Cell. Biol. 55, 129-156 (1998).

5.Giovanni Volpe, Gregory Kozyreff, Dmitri Petrov, "Backscattering position detection for photonic force microscopy,” J. Appl. Phys. 102, 084701 (2007).

6. A. Rohrbach, H. Kress, and E. H. K. Stelzer, "Three-dimensional tracking of small spheres in focused laser beams: influence of the detection angular aperture," Opt. Lett. 28, 411-413 (2003).

Proc. of SPIE Vol. 7762 77622Q-10 